\title{
QCD with domain wall quarks
}

\author{
T. Blum* and A. Soni ${ }^{\dagger}$ \\ Department of Physics, Brookhaven National Laboratory, Upton, NY 11973
}

We present lattice calculations in QCD using a variant of Kaplan fermions [1,2] which retain the continuum $\mathrm{SU}(\mathrm{N})_{\mathrm{L}} \times \mathrm{SU}(\mathrm{N})_{\mathrm{R}}$ chiral symmetry on the lattice in the limit of an infinite extra dimension. In particular, we show that the pion mass and the four quark matrix element related to $K_{0}-\bar{K}_{0}$ mixing have the expected behavior in the chiral limit, even on lattices with modest extent in the extra dimension, e.g. $N_{s}=10$.

\begin{abstract}
A fundamental property of the QCD Lagrangian is chiral symmetry, or invariance under independent global flavor rotations of the left handed and right handed quark fields. Through the familiar Goldstone theorem, the spontaneous breakdown of this symmetry generates massless excitations, and hence chiral symmetry is important to the dynamics of QCD. In reality the symmetry is also explicitly broken by small quark masses, so the excitations are not massless, but very light compared to masses of other hadrons. Until now, lattice simulations of QCD have only accounted for this symmetry approximately or not at all, relying on the continuum limit to restore it exactly. Since most simulations are far from the continuum limit, this is not satisfactory.

The two popular discretizations of the Dirac action used to date for lattice simulations of QCD are the Wilson quark action and the Kogut-Susskind action. For non-zero lattice spacing, each breaks the continuum $\mathrm{SU}(\mathrm{N})_{\mathrm{L}} \times \mathrm{SU}(\mathrm{N})_{\mathrm{R}}$ chiral symmetry. The Wilson action includes a mass-like term, and the Lagrangian quark mass renormalizes additively. In this
\end{abstract}

\footnotetext{
*email: tblum@penguin.phy.bnl.gov

†email: soni@penguin.phy.bnl.gov
} 
case, the chiral symmetry breaking is severe, and fine tuning of the bare parameters is required to obtain a massless pion, which defines the chiral limit. Indeed, these Goldstone bosons may not even be related to chiral symmetry in the nonperturbative regime, but instead to the spontaneous breakdown of parity-flavor symmetry [3]. In addition, Wilson fermions lead to mixing of operators of differing chirality which is absent in the continuum. Physical results depend crucially on removal of these effects. This has been a long-standing problem in lattice calculations of weak matrix elements with light hadrons [4,5] that use Wilson quarks. Weak coupling perturbation theory is unable to accurately match the lattice operators to their continuum counterparts. Various methods have been developed over the years to deal with this problem, and so far none is very satisfactory [6] 9 .

Kogut-Susskind fermions retain an exact $\mathrm{U}(1) \times \mathrm{U}(1)$ remnant of the continuum $\mathrm{SU}(\mathrm{N})_{\mathrm{L}} \times \mathrm{SU}(\mathrm{N})_{\mathrm{R}}$ chiral symmetry [10, so the bare quark mass renormalizes multiplicatively, and the chiral limit is obtained as in the continuum, by taking the quark mass to zero. Because of this remnant chiral symmetry, Kogut-Susskind quarks are preferred for light hadron matrix element calculations and simulations of QCD at non-zero temperature where it is now widely believed that QCD undergoes a phase transition that restores chiral symmetry. This phase transition happens at a critical temperature of roughly $150 \mathrm{MeV}$, or approximately the mass of the pion. Thus, the correct number of pions is important to describe the dynamics of the phase transition. However, at finite lattice spacing the Kogut-Susskind action has only one Goldstone pion instead of the $\mathrm{N}^{2}-1$ in the continuum theory.

For these reasons it is desirable to use a discretization of the QCD action that preserves chiral symmetry. Some time ago Kaplan [1] proposed a discretization of the Dirac action for chiral fermions that avoids the notorious fermion doubling problem and hence could be used to formulate a nonperturbative chiral gauge theory. The impossibility of formulating such a theory that is local and gauge invariant is summarized by the Nielsen-Ninomiya no-go theorem [1]. For each chiral fermion that one starts with in the continuum, a doubler of the opposite handedness appears that renders the lattice theory vector-like. This phenomenon is 
quite general and occurs already in free field theory. However, for free Wilson-like fermions in $\mathrm{d}+1$ dimensions, Kaplan showed that including a mass term with a defect along the extra dimension results in a single chiral fermion which is bound to the d-dimensional defect. Thus a low energy effective theory of chiral fermions may be described on the lower dimensional subspace of the defect. On a finite lattice with periodic boundary conditions, an anti-defect appears with a bound chiral fermion of opposite chirality, so again the theory is vector-like. However, if the extent of the extra dimension is large, the fermions have exponentially small overlap and do not mix; one is free to study the lattice chiral fermion on either wall. Sadly, when the chiral symmetry is gauged, the fermions mix, or if only one is gauged, new particles appear in the spectrum, and the chiral properties are probably lost [12]. But, see also the overlap formalism of Ref. [13].

The above considerations are irrelevant for QCD which is a vector gauge theory, i.e. right and left handed fermions couple to the gauge fields with equal strength. The important point is that Kaplan's fermions retain the full $\mathrm{SU}(\mathrm{N})_{\mathrm{L}} \times \mathrm{SU}(\mathrm{N})_{\mathrm{R}}$ continuum chiral symmetry on the lattice which makes them an attractive alternative for simulating QCD. Actually, at tree level one can show that the symmetry is broken explicitly by terms which are exponentially small in the size of the extra dimension [2]. The main purpose of this work is to verify that these terms remain small in nonperturbative simulations and do not spoil the chiral behavior of observables.

Below we describe calculations in QCD with a variant of Kaplan's original fermions that has been proposed by Shamir [2,14]. In this version only half of the lattice in the extra dimension is used. The chiral fermions, one left handed and one right handed, reside on opposite boundaries of the five dimensional space, and they interact with four dimensional gauge field configurations which are the same on each slice in the five dimensional world. The gauge links in the fifth dimension are set to the identity except for the links which connect the boundaries. Those links explicitly couple the two chiral fermions with a strength equal to $-m$. In perturbation theory it was shown in Ref. [2] that $m$ is proportional to the bare quark mass of the vector fermion constructed from the left handed and right handed quarks 
on the opposite walls of the fifth dimension, $m_{q}=m M(2-M)$. Here $M$ is the ordinary Dirac mass in lattice units for a five dimensional fermion. Then in Ref 15 it was shown that as the number of lattice sites in the extra dimension, $N_{s}$, is sent to infinity, the axial currents of domain wall fermions satisfy nonperturbative Ward identities. Thus the chiral limit is obtained by simply setting $m=0$, unlike in the case of regular Wilson fermions.

For details on Kaplan fermions and the variant of Shamir we refer the reader to Refs. [1,2,15]; here we simply state the quark action for one of the flavors [15].

$$
\begin{aligned}
S_{q} & =-\sum_{x, y, s, s^{\prime}} \bar{\psi}\left(D_{x, y} \delta_{s, s^{\prime}}+\not D_{s, s^{\prime}} \delta_{x, y}\right) \psi \\
\not_{x, y}= & \frac{1}{2} \sum_{\mu}\left(\left(1+\gamma_{\mu}\right) U_{x, \mu} \delta_{x+\hat{\mu}, y}+\left(1-\gamma_{\mu}\right) U_{y, \mu}^{\dagger} \delta_{x-\hat{\mu}, y}\right)+(M-4) \delta_{x, y} \\
\not D_{s, s^{\prime}} & = \begin{cases}\frac{\left(1+\gamma_{5}\right)}{2} \delta_{1, s^{\prime}}-m \frac{\left(1-\gamma_{5}\right)}{2} \delta_{N_{s}-1, s^{\prime}}-\delta_{0, s^{\prime}} & s=0 \\
\frac{\left(1+\gamma_{5}\right)}{2} \delta_{s+1, s^{\prime}}+\frac{\left(1-\gamma_{5}\right)}{2} \delta_{s-1, s^{\prime}}-\delta_{s, s^{\prime}} & 1 \leq s \leq N_{s}-2 \\
-m \frac{\left(1+\gamma_{5}\right)}{2} \delta_{0, s^{\prime}}+\frac{\left(1-\gamma_{5}\right)}{2} \delta_{N_{s}-2, s^{\prime}}-\delta_{N_{s}-1, s^{\prime}} & s=N_{s}-1\end{cases}
\end{aligned}
$$

where $\psi$ is a five dimensional Dirac fermion. The $U_{x, \mu}$ are the usual four dimensional gauge links and are elements of the color group SU(3). Note the Wilson term which removes the doublers is added to the action with minus the conventional sign. $x$ and $y$ denote four dimensional coordinates while $s$ and $s^{\prime}$ denote coordinates along the extra fifth dimension. $N_{s}$ is the number of sites in this dimension. The simplest choice for operators which create and destroy light four dimensional quarks are 15

$$
\begin{aligned}
& q_{x}=\frac{\left(1+\gamma_{5}\right)}{2} \psi_{x, 0}+\frac{\left(1-\gamma_{5}\right)}{2} \psi_{x, N_{s}-1}, \\
& \bar{q}_{x}=\bar{\psi}_{x, N_{s}-1} \frac{\left(1+\gamma_{5}\right)}{2}+\bar{\psi}_{x, 0} \frac{\left(1-\gamma_{5}\right)}{2} .
\end{aligned}
$$

In fact, any operators localized near the boundaries that have finite overlap with the boundary states will do as interpolating operators for the quark states.

As a first step in implementing domain wall quarks for QCD, we measure observables constructed from the above operators on existing configurations. The gauge field configurations that we have used are a set of 20 lattices generated with two flavors of Kogut-Susskind 
dynamical quarks at gauge coupling $6 / g^{2}=5.7$ and bare quark mass $m_{K S}=0.01$ [16]. The lattice size for these configurations is $16^{3} \times 32$. In all of our calculations, the Dirac mass $M$ was set to 1.7 .

For free quarks one must take $0<M<1$ to obtain massless states with strictly positive Green's functions [2]. For $1<M<2$ there exists a massless mode, but its Green's function oscillates like $(1-M)^{s}$. This free field condition is renormalized at strong coupling as we found only heavy states for $M<1$. This can be seen from perturbative arguments [2] and a simple mean field treatment which gives $4-4 U_{0}<M<6-4 U_{0}$ [17]. Here $U_{0}$ is the fourth root of the plaquette expectation value, or the mean field value of the gauge link. The mean field propagator then falls off like $\left(5-\left(M+4 U_{0}\right)\right)^{s}$. On the above configurations $U_{0} \approx 0.577$, yielding $M \approx 1.52$ for the most rapid decay of the propagator in the extra dimension, and thus the smallest overlap of the boundary states. The above mean field result agrees fairly well with the "optimal" value of $M=1.7$ which we determined by observing the exponential decay of the quark propagator in the extra dimension. As $M$ departs from this optimal value, the extra dimension must be increased to avoid possible overlap of the massless modes. While massless pions may still exist for $M<1$ at strong coupling, we did not observe them due to the relatively small extent of the lattice in the extra dimension. Thus, for efficient lattice simulations with domain wall quarks it is important to determine the optimal value of $M$. In this study we set the number of sites in the fifth direction to $N_{s}=4$ and 10 to study the effects of finite size in the extra dimension on the chiral symmetry properties of observables. Below we will see that for $M=1.7, N_{s}=10$ is sufficiently large to suppress the overlap of the light modes.

The simplest test of chiral symmetry (breaking) for domain wall fermions is to measure the pseudo-scalar two-point correlation function and extract the mass. Chiral perturbation theory requires this mass to go to zero like $\sqrt{m}$, or $m_{\pi}^{2} \propto m$. In Fig. 11 we show $m_{\pi}^{2}$ versus $m$ for the two values of $N_{s}$. The masses were extracted from a single particle covariant fit to the pseudo-scalar correlator at large Euclidean time separation from the source (see below). At $N_{s}=10$, the lightest two points linearly extrapolate to $0.0002 \pm 0.0160$ while for $N_{s}=4$ 
the results clearly miss the origin to the left. It appears that $N_{s}=10$ is large enough to suppress the explicit chiral symmetry breaking effects due to the overlap of the two chiral modes. The $N_{s}=4$ case shows that the approach to the $N_{s} \rightarrow \infty$ limit is from above which is expected since the Wilson term is added to the action with minus the conventional sign.

For a more stringent test of the chiral symmetry properties of domain wall quarks we turn to the calculation of hadronic matrix elements, which as mentioned above is problematic with conventional lattice operators. The canonical example is the $K_{0}-\bar{K}_{0}$ mixing matrix element, $M_{L L}$, required to determine the $C P$ violating phase of the CKM matrix. Explicitly, $M_{L L}=$ $\left\langle\bar{K}\left|\left[\bar{s} \gamma_{\nu}\left(1-\gamma_{5}\right) d\right]^{2}\right| K\right\rangle$ and is expected to vanish as $m_{K}^{2}$ in the chiral limit. However, as we have mentioned, it is well known that the matrix element of this operator constructed from Wilson quarks does not vanish in the chiral limit. Only by fine tuning a linear combination of all four quark operators with differing Dirac structures does one obtain the correct behavior. To date, no satisfactory method of fine tuning has been found to solve this problem [6 9], so lattice operators which avoid this mixing are much preferred, cf. Kogut-Susskind quarks and now domain wall quarks.

In Fig. 2 we show $\left\langle\bar{K}\left|\left[\bar{s} \gamma_{\nu}\left(1-\gamma_{5}\right) d\right]^{2}\right| K\right\rangle /\left|\left\langle 0\left|\bar{s} \gamma_{5} d\right| K\right\rangle\right|^{2}$ versus $m$ for both values of $N_{s}$ and the usual Wilson quark operator whose mixing coefficients were determined using lattice weak coupling perturbation theory [18,19] with a boosted coupling [20]. This ratio has the same mass dependence as $M_{L L}$ in the chiral limit since the denominator is just proportional to the square of the kaon decay constant. From Fig. 2 it is clear that the $N_{s}=10$ result exhibits the required behavior in the chiral limit: the values at the two smallest quark masses extrapolate linearly to $.002 \pm .015$ at $m=0$. On the other hand, the $N_{s}=4$ domain wall quark and Wilson quark results significantly miss the origin. We note that while the errors are considerably larger for the Wilson case, the results at each quark mass are highly correlated and a fit which accounts for this does not go near the origin. Indeed, previous lattice calculations have shown that increased statistics do not remedy the problem. Also note that the domain wall results again approach the large $N_{s}$ limit from above.

The ratios in Fig. 2 corresponding to domain wall quarks are determined with the follow- 
ing procedure. First we calculate five dimensional propagators from wall sources at times 0 and 31 (the ends of the lattice) on each boundary in the fifth dimension. The quark propagators are then constructed using Eq. (2). Because of the chirality projection operators in Eq. (2), only two source spins on each boundary are required. We find that the added cost of inverting the five dimensional fermion matrix scales linearly with $N_{s}$. Next, these propagators were contracted using point sinks to form three correlation functions on each gauge field configuration, a two-point pseudo-scalar correlation function from each wall source and a three-point correlation function from the same two wall sources. Each correlation function is averaged over configurations. The ratio is found by dividing the three-point correlation function by the product of the pseudo-scalar correlators, all three being evaluated at the same sink time slice. For large time separations between the sink time slice and the two wall sources, the result is a constant. Our results were averaged over sink time slices 10 to 20 where this constant plateau was evident. The errors displayed in Fig. (2), which are statistical, are calculated from a jackknife procedure. In fact, simultaneous covariant fits to all three correlation functions, which have good $\chi^{2}$, give similar results. The Wilson quark results were calculated in similar fashion, but with point sources.

A similar ratio as shown in Fig. 2, but with $\left\langle 0\left|\bar{s} \gamma_{5} d\right| K\right\rangle$ replaced by $\left\langle 0\left|\bar{s} \gamma_{5} \gamma_{0} d\right| K\right\rangle$, gives the parameter $B_{K}$ which chiral perturbation theory predicts to be non-zero in the chiral limit. We are in the process of calculating this important phenomenological quantity. Preliminary measurements indicate that the ratio depends weakly on $m$ and is non-zero as $m \rightarrow 0$. The numerical value of $B_{K}$ is scale dependent (as is $M_{L L}$ ) and thus requires a renormalization factor. In addition, finite lattice spacing effects must be studied by simulating at several couplings to understand the continuum limit of $B_{K}$. Thus much work needs to be done before a meaningful value can be given.

The above results show that domain wall quarks exhibit excellent chiral behavior, even at $g^{2} \sim 1$. In particular the bare quark mass appears to receive only multiplicative renormalization, i.e. no fine tuning of the couplings is required to reach the chiral limit, in contrast to the case with Wilson quarks. Strictly speaking this is true only in the limit $N_{s} \rightarrow \infty$; 
however, residual chiral symmetry breaking effects coming from finite $N_{s}$, which were not detectable in our calculations with $N_{s}=10$, are in principle removable by simply increasing $N_{s}$. These encouraging results also show that domain wall quarks are a vast improvement over Wilson quarks for matrix element calculations. Again, there is no fine tuning or cumbersome operator subtractions necessary to obtain the correct chiral behavior. Similarly, they are likely to prove useful for other QCD calculations where chiral symmetry is crucial, e.g. the QCD phase transition for non-zero temperature where current simulations suffer from explicit chiral symmetry breaking effects. Finally, we re-emphasize that domain wall quarks at $N_{s}=10$ retain the full chiral symmetry of continuum QCD to a remarkable degree, unlike either Wilson or Kogut-Susskind quarks.

We thank Y. Shamir and M. Creutz for suggesting the use of domain wall fermions for matrix element calculations and M. Creutz for helpful discussions. Our domain wall fermion code relies heavily on the four dimensional MILC code [21], which we gratefully acknowledge. This research was supported by US DOE grant DE-AC0276CH0016. The numerical computations were carried out on the NERSC T3D and the SDSC Paragon.

[1] D. Kaplan, Phys. Lett. B288, 342 (1992).

[2] Y. Shamir, Nucl. Phys. B406, 90 (1993).

[3] S. Aoki, Nucl. Phys. B314, 79 (1989); S. Aoki, A. Ukawa, and T. Umemura, Phys. Rev. Lett. 76, 873 (1996); S. Aoki and A. Gocksch, Phys. Rev. D45, 3845 (1992); M. Creutz, "Wilson fermions at finite temperature", hep-lat/9608024.

[4] C. Bernard et al. , Phys. Rev. Lett. 55, 2770 (1985).

[5] See A. Soni, Nucl. Phys. B (Proc.Suppl.) 47, 43 (1996), and references therein.

[6] M.B. Gavela, et al. , Nucl. Phys. B 306, 677 (1988). 
[7] C. Bernard and A. Soni, Nucl. Phys. B (Proc.Suppl.) 9, 155 (1989).

[8] R. Gupta, et al. , Phys. Rev. D47, 5113 (1993); R. Gupta, et al. , "Matrix elements of 4-fermion operators with quenched Wilson fermions", hep-lat/9611023.

[9] G. Martinelli, et al. , Nucl. Phys. B445, 81 (1995); S. Aoki, et al. , " $B_{K}$ with the Wilson quark action: a non-perturbative resolution of operator mixing using chiral Ward identities", hep-lat/9608150.

[10] N. Kawamoto and J. Smit, Nucl. Phys. B192, 100 (1981).

[11] H.B. Nielsen and M. Ninomiya, Nucl. Phys. B185, 20 (1981); L. Karsten and J. Smit, Nucl. Phys. B183, 103 (1981).

[12] M. Golterman, et al. , Phys. Rev. D49, 1606 (1994); M. Golterman and Y. Shamir, Phys. Rev. D51, 3026 (1995); Phys. Lett. B353, 84 (1995); Erratum Phys. Lett. B359, 422 (1995).

[13] R. Narayanan and H. Neuberger, Phys. Rev. Lett. 71, 3251 (1993); Nucl. Phys. B412, 574 (1994); Nucl. Phys. B (Proc.Suppl.) 34, 587 (1994); R. Narayanan, Nucl. Phys. B (Proc.Suppl.) 34, 95 (1994); R. Narayanan and H. Neuberger, Nucl. Phys. B443, 305 (1995). Note that the overlap formalism can also be used to study vector gauge theories with chiral symmetry. However, numerically it is expected to be quite difficult to implement for QCD.

[14] Recently, domain wall fermions have also been used in simulations of the $2+1$ dimensional Schwinger model. See P. Vranas, "Domain wall fermions and MC simulations of vector theories", hep-lat/9608078; A. Jaster, "Simulating the massive Schwinger model with chiral defect fermions", hep-lat/9605011.

[15] Y. Shamir and V. Furman, Nucl. Phys. B439, 54 (1995).

[16] The configurations are a subset of lattices generated by the Columbia group to whom we are indebted for their use. See F. Brown, et al. , Phys. Rev. Lett. 67, 1062, (1991).

[17] We thank one of the referees for pointing out this simple argument. 
[18] G. Martinelli, Phys. Lett. B 141, 395 (1984).

[19] C. Bernard, T. Draper, and A. Soni, Phys. Rev. D36, 3224 (1987).

[20] G.P. Lepage and P. Mackenzie, Phys. Rev. D. 48, 2250 (1993).

[21] C. Bernard, T. DeGrand, C. DeTar, S. Gottlieb, A. Krasnitz, M.C. Ogilvie, R.L. Sugar, and D. Toussaint, in Workshop on Fermion Algorithms, edited by H. J. Hermann and F. Karsch, (World Scientific, Singapore, 1991). 


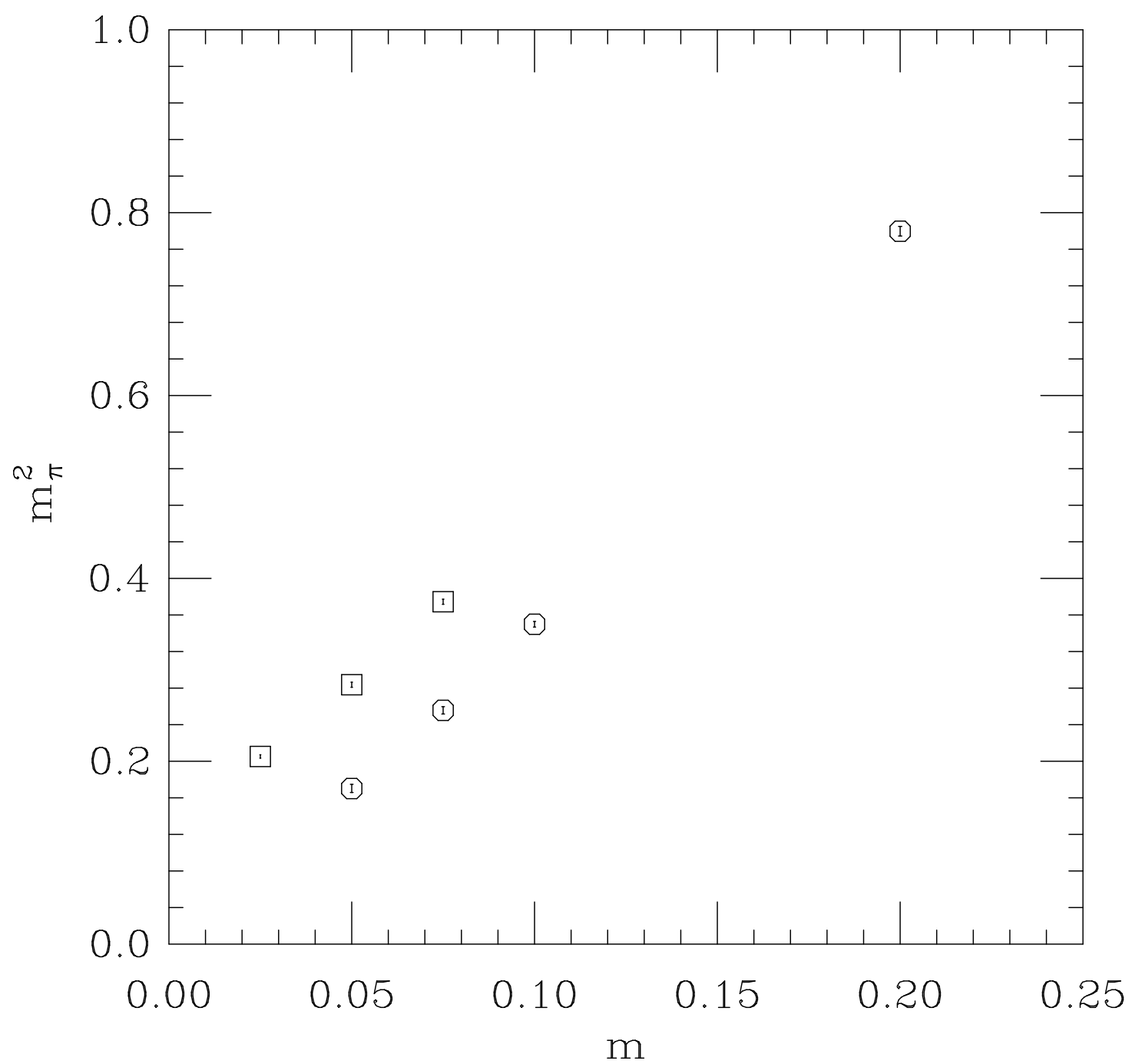

FIG. 1. The pion mass squared as function of $m . m$ is proportional to the quark mass. The last two points for $N_{s}=10$ (octagons) extrapolate linearly to zero well within statistical errors. The squares denote results for $N_{s}=4$. 


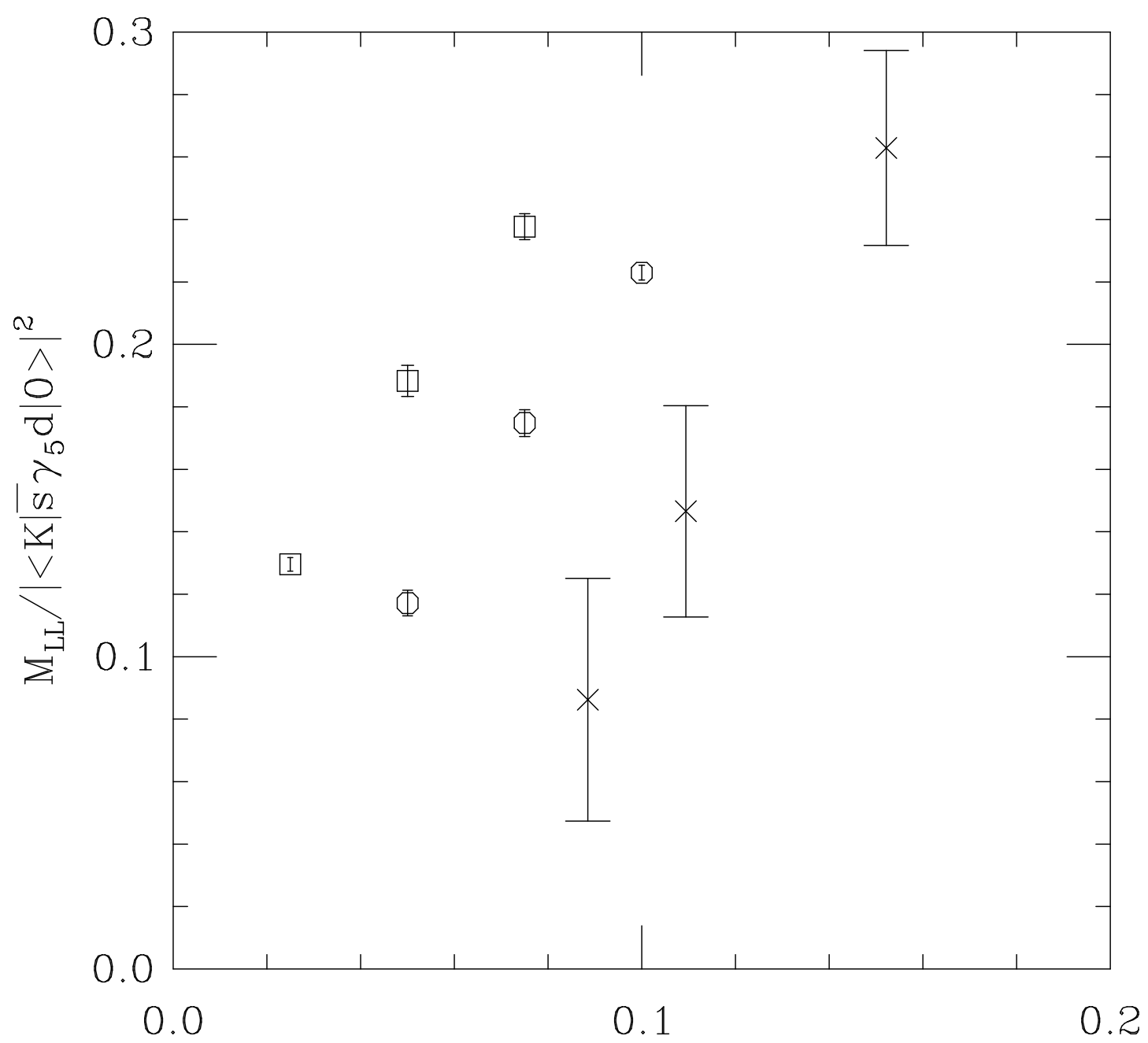

$\mathrm{m}$

FIG. 2. The ratio of the four quark matrix element for $K_{0}-\bar{K}_{0}$ mixing to the square of the pseudo-scalar density matrix element, calculated with domain wall fermions $\left(\operatorname{octagons}\left(N_{s}=10\right)\right.$ and squares $\left.\left(N_{s}=4\right)\right)$. The $N_{s}=10$ curve exhibits the correct behavior in the chiral limit. Also shown is the result using the same gauge field configurations for Wilson quarks (crosses) which extrapolates to zero far from $m=0$ (note that for Wilson quarks the quark mass is defined as the difference of the inverse quark hopping parameter with the inverse critical hopping parameter, $\left.m \equiv \frac{1}{2}\left(\kappa^{-1}-\kappa_{c}^{-1}\right)\right)$. 